\title{
Improved Windowed Cross Wigner-Ville Distribution for PSK Class Signals
}

\author{
Chee Yen Mei and Ahmad Zuri Sha'ameri
}

\begin{abstract}
Instantaneous information bearing phase is an important parameters in the analysis of a digitally phase modulated signal. Cross time-frequency distributions (XTFD) have been proposed recently to represent the class of Phase Shift Keying (PSK) signals. However, due to the presence of duplicated terms, the estimation of the instantaneous bearing phase is inaccurate. A fixed lag window width can be used as a kernel but it is insufficient to completely remove the duplicated terms. Here, a new improved windowed cross Wigner-Ville distribution (XWVD) is proposed as a method to obtain a better cross time-frequency representation (XTFR) for binary phase shift keying (BPSK) and quadrature phase shift keying (QPSK) signals. By adaptively adjusting the lag window width, the duplicated terms are removed and an accurate representation of the cross TFR are obtained at low SNR conditions, as low as 3dB.
\end{abstract}

Index Terms-Cross time-frequency analysis, cross Wigner-Ville distribution, instantaneous phase, instantaneous information bearing phase.

\section{INTRODUCTION}

As the demand for faster and more secure communication services increases, many communication applications have moved from analogue to digital. Spectrum is a scarce resource, thus the phase shift keying (PSK) modulation such as binary phase shift keying (BPSK) and quadrature phase shift keying (QPSK) and M-ary PSK have been frequently used. The phase modulation is more robust to noise and more bandwidth efficient [1]. Signal analysis and classification is an intermediate step between signal detection and information recovery. It plays an important role in many applications such as spectrum monitoring systems, electronic surveillance, electronic warfare and electronic measurement equipments [2]. Various methods had been proposed for the analysis and classification of digital modulation signal over the past decades [3]-[5]. Generally, the method used can be classified into two: decision theoretic and pattern recognition method. The decision theoretic method is based on a likelihood function with some priori knowledge of the signal. On the other hand, for the pattern recognition method, it first performs estimation of the signal parameters and then classifies the signal according to the parameters obtained. All signals are non-stationary in nature, thus time-frequency

Manuscript received April 10, 2012; revised June 15, 2012.

Y. M. Chee is with the Microelectronics \& Computer Engineering Department, Universiti Teknologi Malaysia, 81310 Skudai, Johor, Malaysia. (e-mail: est26cym@yahoo.com).

A. Z. Sha'ameri is with Microelectronics \& Computer Engineering Department, Universiti Teknologi Malaysia, 81310 Skudai, Johor, Malaysia. (e-mail:zuri@fke.utm.my). analysis method is one of the useful tools to completely characterize the signals.

There are numerous works done on the instantaneous frequency (IF) estimation [6]-[8] as IF is an important parameter in many applications such as radar, sonar, communication and biomedicine. The authors in [8], proposed a method to estimate the IF of a linear FM signals from the peaks of the time-frequency distribution (TFD). Works on bilinear TFD [9] can give information on the IF of a signal and it works well at very low SNR - as low as $2 \mathrm{~dB}$ - at the loss of phase information. Thus, there is a need to find a TFD that can accurately represents the instantaneous information bearing phase which was useful to analyze PSK signals.

Instantaneous phase (IP) [8] of a signal is defined as

$$
\phi(t)=\omega t+\varphi(t)
$$

where $\omega$ is the angular frequency and $\varphi(t)$ is the instantaneous information bearing phase. The IF of a signal can be obtained from the first derivative of the IP. Signals that we deal with are phase modulated signal, our interest in this paper is to find the instantaneous information bearing phase $\varphi(t)$ of the signal. A new approach was proposed using cross TFD (XTFD) to represent PSK signals in [10], but the presence of duplicated terms in the time-frequency representation (TFR) results in the inaccurate estimation of the instantaneous information bearing phase [10]. In this paper, an improved XTFD is proposed which gives better accuracy in the instantaneous information bearing phase estimation of PSK signal. Signals parameters such as the carrier frequency, symbol duration, instantaneous energy and instantaneous information bearing phase can be obtained from the cross time-frequency representation (XTFR).

\section{II.SIGNAL MODELS}

In this paper, the signal of interest is digitally phase modulated signal. For an arbitrary BPSK or QPSK signals, it can be represented as

$$
z(t)=A \sum_{k=1}^{N-1} \exp \left(j 2 \pi f_{k}\left(t-k T_{b}\right)+\varphi_{k}(t)\right) \Pi\left(t-k T_{b}\right)
$$

where $k$ represents the order of the binary sequence transmitted, $A$ represents the signal amplitude, $f_{k}$ is the subcarrier frequency, $\varphi_{k}$ represents the instantaneous information bearing phase and $T_{b}$ is the symbol duration of 
the signals. Here $A$ and $f_{k}$ are constant as the signal considered are PSK signals.

The box function, $\Pi(t)$ is defined as

$$
\begin{aligned}
\Pi(t) & =1 \text { for } 0 \leq t \leq T_{b} \\
& =0 \text { elsewhere }
\end{aligned}
$$

The signal parameters used in this paper are as below:

$$
\begin{aligned}
& \begin{array}{l}
\text { BPSK1 }: f_{0}=1000, T_{b}=20 m s \\
\text { BPSK2 }: f_{0}=1000, T_{b}=10 m s
\end{array}, \varphi_{k}=\left\{\begin{array}{l}
\pi \text { for } \mathrm{s}=1 \\
0 \text { for } \mathrm{s}=0
\end{array}\right.
\end{aligned}
$$

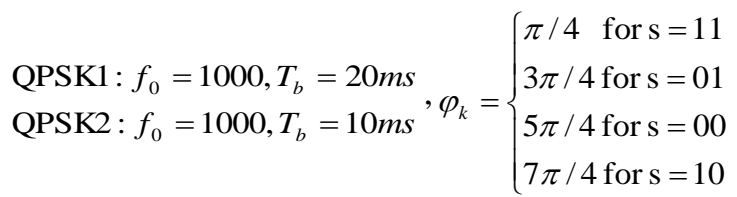

\section{CRoss Wigner Ville Distribution}

The XTFD is related to the cross correlation function where two different signals are used to obtain its cross bilinear product and it can be derived from the bilinear TFD [10]. The general formulation for the XTFD, formed between an analytical signal, $z(t)$ and a reference, $r(t)$, can signal be expressed as

$$
\rho_{z r}(t, f)=\int_{-\infty}^{\infty} G(t, \tau) \underset{(t)}{*} K_{z r}(t, \tau) \exp (-j 2 \pi f \tau) d \tau
$$

where $K_{z r}(t, \tau)$ is the cross bilinear product and $G(t, \tau)$ is the separable kernel function, both are given by (5) and (6) respectively.

$$
\begin{gathered}
K_{z r}(t, \tau)=z\left(t+\frac{\tau}{2}\right) r *\left(t-\frac{\tau}{2}\right) \\
G(t, \tau)=H(t) w(\tau)
\end{gathered}
$$

The kernel functions for the windowed cross Wigner-Ville distribution (WXWVD) are defined as

$$
G(t, \tau)=\delta(t) w(\tau)
$$

The lag window function $w(\tau)$ can be any window function used in filter design or spectrum analysis. The WXWVD can be represented as in (8)

$$
\rho_{z r}(t, f)=\int_{-\infty}^{\infty} K_{z r}(t, \tau) w(\tau) \exp (-j 2 \pi f \tau) d \tau
$$

\section{CRoss BILINEAR PRODUCT}

The cross bilinear product represents a signal in the time-lag domain and it has both auto terms and duplicated terms. The duplicated term is used here to represent the unwanted term rather than cross term which usually appears in bilinear TFD [9]. The use of different notation for the unwanted terms is because the duplicated terms carries the same information as the auto terms but shifted in time and lag domain. For any digital phase modulation signal the time-lag representation of the cross bilinear products are defined as

$$
K_{z r, \text { auto }}=\sum_{k=1}^{N-1}|A|^{2} \exp \left(j\left(2 \pi f_{k} \tau+\phi_{k}\right)\right) K_{\mathrm{r}(3)}(k-1) T_{(33 j)}
$$

$$
K_{z r, \text { duplicated }}=\sum_{\substack{k=1 \\
k \neq l}}^{N-1} \sum_{l=1}^{N-1}\left(\begin{array}{l}
|A|^{2} \exp \left(j\left(2 \pi f_{k} \tau+\phi_{k}\right)\right) \times \\
K_{\Pi}\left(t-\frac{(k+l-2) T_{b}}{2}, \tau(l-k) T_{b}\right)
\end{array}\right)
$$

where $K_{\Pi}(t, \tau)$ is the instantaneous autocorrelation function of the box function given as

$$
K_{\Pi}(t, \tau)=\Pi\left(t+\frac{\tau}{2}\right) \Pi\left(t-\frac{\tau}{2}\right)
$$

Shown in Fig. 1 is the time-lag representation of the cross bilinear product, where all the auto terms lie along the $\tau=0$ axis. The windowed cross bilinear product is shown by the shaded area. By introducing a fixed window along the lag axis can removed the duplicated terms that lie far away from the lag axis. However, this is insufficient to completely remove all the duplicated terms. Observed that at time $t=2 T_{b}$, there are both auto terms and duplicated terms exist even after a fixed lag window is employed. Interaction between the auto and the irremovable duplicated terms causes the in the inaccuracy of phase estimation.

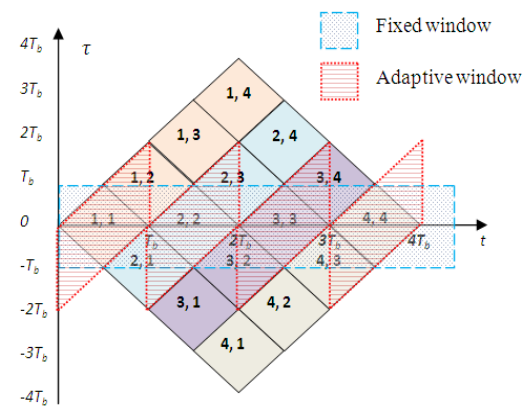

Fig. 1. Cross bilinear product of phase modulated signal with fixed window width $\mathrm{w}(\tau)=T_{\mathrm{b}}$ and adaptive window.

\section{V.ADAPTIVE WINDOWED CROSS WIGNER-VILLE DISTRIBUTION}

Since there are still irremovable duplicated terms by a fixed window as shown in Fig. 1, the phase estimation during the transition period will be inaccurate. This shows that the accuracy in the TFR is greatly dependent on the kernel parameters. In order to have a better phase estimation of the signals, an adaptive window method is introduced in this paper. By employing an adaptive window to the XWVD, most of the duplicated terms that causes interference cross TFR will be removed. This leads to a new TFD known as the adaptive windowed cross Wigner-Ville distribution (AWXWVD) which is defined as

$$
\rho_{z r, A W X W V D}(t, f)=\int_{-\infty}^{\infty} K_{z r}(t, \tau) w(t, \tau) \exp (-j 2 \pi f \tau) d \tau
$$




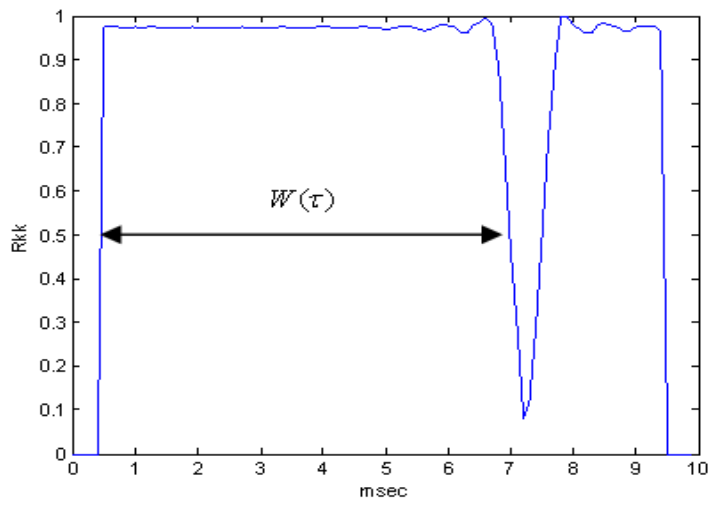

(a)

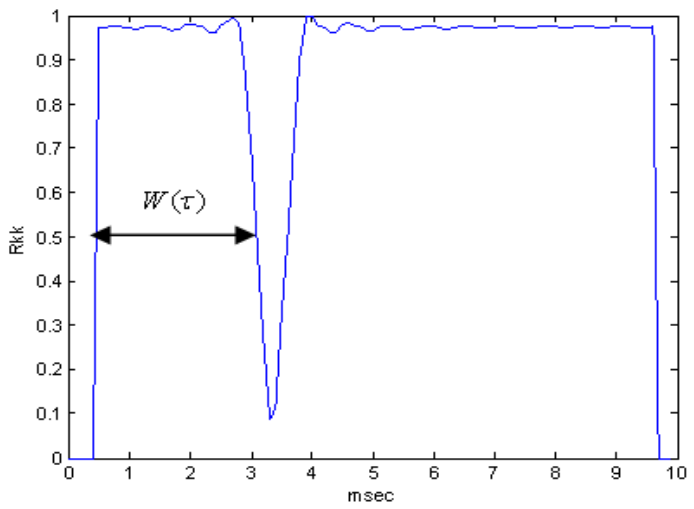

(b)

Fig. 2. Autocorrelation function of the cross bilinear product evaluated at $t=30 \mathrm{~ms}$ (a) positive lag (b) negative lag
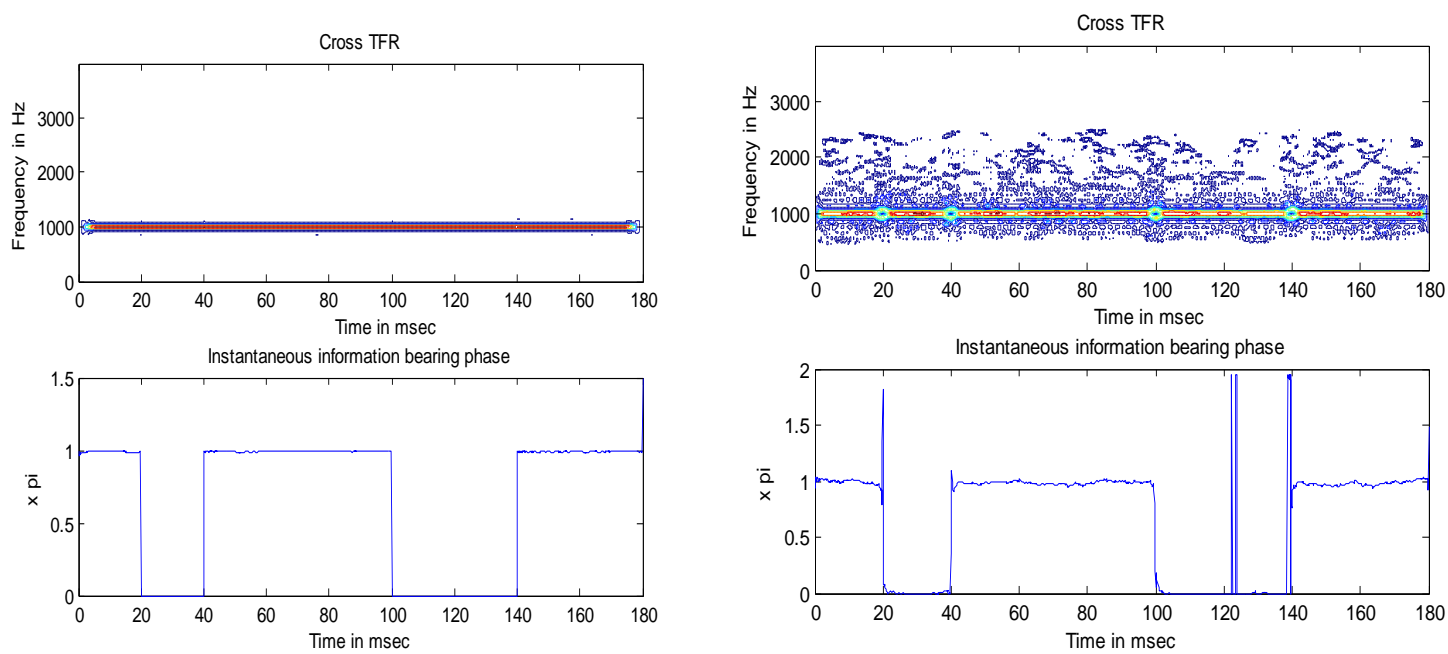

(a) SNR $12 \mathrm{~dB}$

(b) SNR 3dB

Fig. 3. Cross TFR for BPSK1 and its respective instantaneous information bearing phase with SNR (a) 12dB (b) 3dB
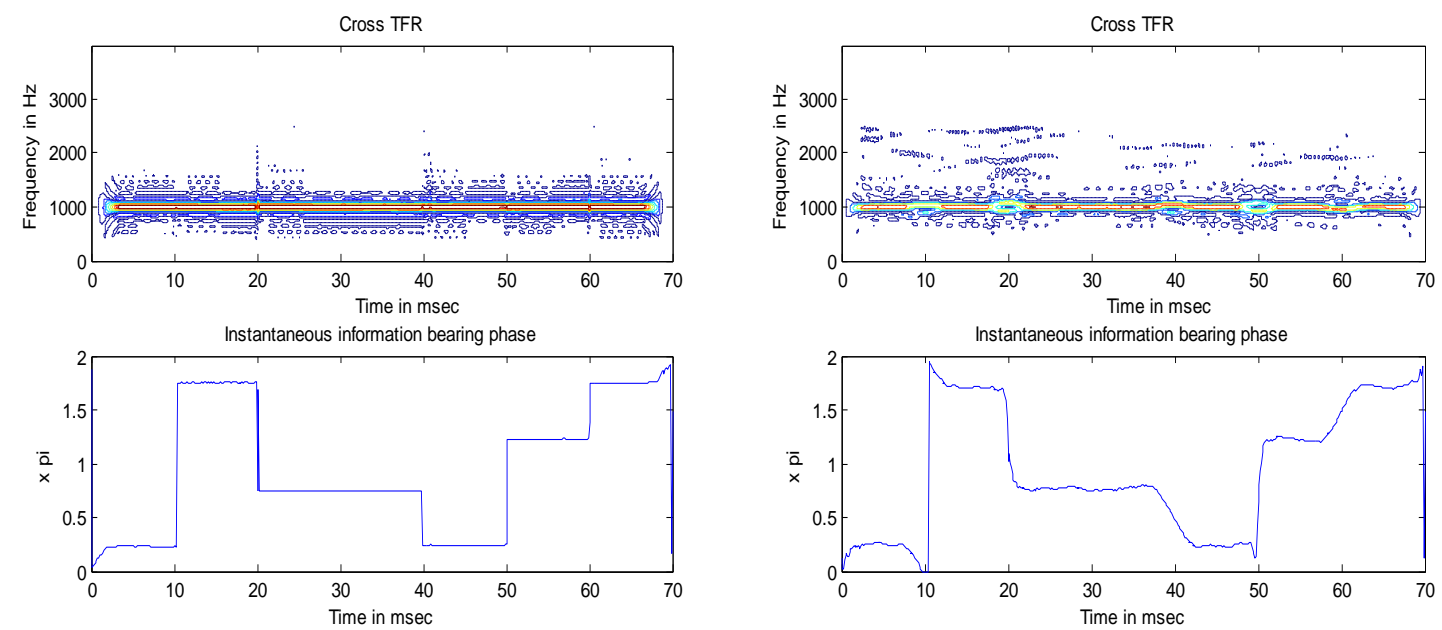

(a) SNR $12 \mathrm{~dB}$

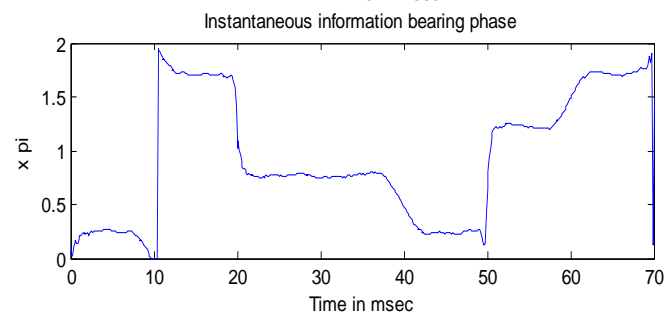

(b) SNR 4dB

Fig. 4. Cross TFR for QPSK1 and its respective instantaneous information bearing phase with SNR (a) $12 \mathrm{~dB}$ (b) $4 \mathrm{~dB}$

where $w(t, \tau)$ is the time-dependent lag window. Since all the auto terms lie along the $\tau=0$ axis, then the window width can be estimated using autocorrelation function at all time instants. By performing the localized lag autocorrelation function at every time instant along the lag axis, the variation of the signals at every step of time can be detected. 
The autocorrelation function can be defined as

$$
R_{K K}(t, \varsigma)=\int_{-T}^{T} w_{a}(\tau)^{2} K_{z r}(t, \tau) K_{z r}(t, \varsigma-\tau) d \tau
$$

where $w_{a}(\tau)$ is the analysis window, $T$ is the signal duration and $\varsigma$ is the lag instant. The analysis window is defined as

$$
w_{a}(\tau)=\frac{1}{\tau_{a}} \quad 0<\tau_{\mathrm{a}}<<T_{b}
$$

The amplitude of the autocorrelation function is used to determine the miscorrelation of the signal in lag and then estimate the window width for each time instants. The amplitude of the autocorrelation function is normalized and the possible range for the normalized autocorrelation function is

$$
0 \leq\left|R_{K K}(t, \varsigma)\right| \leq 1
$$

$\left|R_{K K}(t, \varsigma)\right|=1$ indicates that there is a high correlation of the signal in lag, as the value decreases the correlation becomes less. At lag instant, $\varsigma=0$ the correlation function, $\left|R_{K K}(t, 0)\right|$ should approaches unity as all the auto terms lie along the time axis. Since the cross bilinear product is not symmetry, the adaptive lag window resembled a parallelogram shape as shown in Fig. 1. Shown in Fig. 2 is the autocorrelation function evaluated at time $t=30 \mathrm{~ms}$ in the positive lag and negative lag direction. When there is a drastic drop observed in the plot, this is where the miscorrelation of the signal starts to occur. A threshold value of 0.5 is set to determine the boundary where the window width should be estimated. This threshold value is obtained experimentally using all the test signals defined in Section 2.

\section{RESULTS}

The system is tested for both BPSK and QPSK signals defined in Section 2. In order to validate the performance of the AWXWVD simulation was done in the presence of noise. Additive white Gaussian noise (AWGN) are randomly generated and added to the signal such that the SNR is $\leq$ $12 \mathrm{~dB}$. A slice of the signal at the peak location is analyzed and the instantaneous information bearing phase of the signal is estimated from there. Shown in Fig. 3 is the XTFR and the estimated instantaneous information bearing phase for BPSK1 with different SNR. Fig. 4 is the XTFR and the estimated instantaneous information bearing phase for QPSK1 with different SNR. From Fig. 3 and Fig. 4, it is observed that there is no distortion in information bearing phase during the transition period which appear in [10]. The unwanted components that appear in the XTFR in Fig. 3(b) and Fig. 4(b) is due to the high intensity of noise. For both BPSK1 and BPSK2 signals, the system is capable to give accurate information bearing phase estimation at low SNR (as low as 3dB). On the other hand, for QPSK1 and QPSK2 signals the system works well at SNR as low as $4 \mathrm{~dB}$. Higher minimum SNR required for QPSK signals to produce accurate phase estimation because there are more symbols and all the symbols are located closer to each other compared to the BPSK signal. Therefore, it is more subjected to noise distortion. For both BPSK and QPSK signals, a sharp XTFR were obtained all the time as the window width is adaptively adjusted and optimized by the system.

\section{CONCLUSION}

The proposed method, AWXWVD is an improvement to the previous WXWVD which utilize a fixed window as a kernel. In this paper, it shows that the AWXWVD is capable to give accurate information bearing phase estimation for the PSK class of signals. The system changes the window width and position adaptively to produce a sharp XTFR. Thus, there is no need to manually set the window width. Apart from that, the method proposed is robust to noise and capable to work at SNR as low as $3 \mathrm{~dB}$. Accurate XTFR and phase estimation are crucial as from there, signal parameters were obtained.

\section{ACKNOWLEDGMENT}

The authors would like to thank Ministry of Higher Education (MOHE) Malaysia for its financial support and Universiti Teknologi Malaysia for providing the resources for this research.

\section{REFERENCES}

[1] T. P. Zielinski, "Instantaneous phase shift estimation methods," in Proc. of IEEE Instrumentation and Measurement Technology Conference, 1996, Brussels, Belgium, vol. 1, pp.162-167.

[2] B. Ramkumar, "Automatic modulation classification for cognitive radios using cyclic feature detection," IEEE Circuits and Systems Magazine, 2009, vol. 9, no. 2, pp. 27-45.

[3] W. Dan, G. Xuemai, and G. Qing, "A new scheme of automatic modulation classification using wavelet and WSVM," in Proc. of IEEE 2nd International Conference on Mobile Technology, Applications and Systems, Guangzhou, China, 2005, pp. 5-10.

[4] J. Yuan, Z. Y. Zhang, and P. L. Qiu, "Modulation classification of communication signals," in Proc. of IEEE Military Communications Conference, Monterey, CA , 2004, vol. 3, no., pp. 1470- 1476.

[5] L. L. Meng and X. J. Si, "An improved algorithm of modulation classification for digital communication signals based on wavelet transform," in Proc.of IEEE International Conference on Wavelet Analysis and Pattern Recognition, Beijing, China, 2007, vol. 3, no., pp.1226-1231.

[6] K. V. S. Narayana and T. V. Sreenivas, "Comparison of IF estimation methods for noise robustness," in Proc. of IEEE Region 10 Conference (TENCON), Hyderabad, India, 2008, pp. 1-6.

[7] E. Sejdic, I. Djurovic, and L. Stankovic, "Quantitative Performance Analysis of Scalogram as Instantaneous Frequency Estimator," IEEE Trans. on Signal Processing, vol. 56, no. 8, pp. 3837-3845, Aug. 2008.

[8] B. Boashash, "Estimating and interpreting the instantaneous frequency of a signal. II. Algorithms and applications," in Proc. of the IEEE, vol 80, no. 4, pp. 540-568, 1992.

[9] J. L. Tan, A. Z. B. Sha'ameri, "Adaptive optimal kernel smooth-windowed Wigner-Ville bispectrum for digital communication signals," Signal Processing, vol. 91, no. 4, pp. 931-937, April 2011.

[10] Y. M. Chee and A. Z. Sha'ameri, "Use of the cross time-frequency distribution for the analysis of the class of PSK signals," in Proc. of IEEE International Conference on Computer and Communication Engineering (ICCCE), Kuala Lumpur, Malaysia, 2010, pp.1-5. 


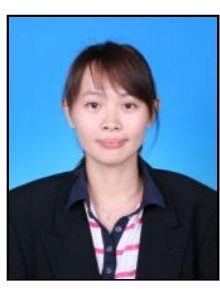

Chee Yen Mei was born in Kuala Lumpur, Malaysia in 1985.She received the B.E. degree in electrical (telecommunication) engineering from Universiti Teknologi Malaysia (UTM), Johor, Malaysia in 2009. She is currently working towards the Ph.D. degree at Digital Signal Processing Research Laboratory, Universiti Teknologi Malaysia. Her research interests include time-frequency signal analysis, signal analysis and classification, and signal processing in telecommunication. Ms. Chee is a Graduate Student Member of the IEEE.

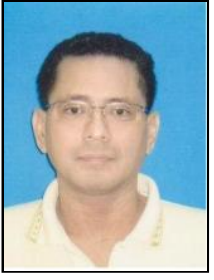

Ahmad Zuri bin Sha'ameri obtained his B. Sc. in Electrical Engineering from the University of Missouri-Columbia, USA in 1984, and M. Eng. Electrical Engineering and $\mathrm{Ph} \mathrm{D}$ both from Universiti Teknologi Malaysia (UTM) in 1991 and 2000 respectively. At present, he is the Coordinator for Digital Image and Signal Processing (DSIP) Research Group, Infocomm Research Alliance and Coordinator for the DSP Research Lab, Faculty of Electrical Engineering, UTM. His research interest includes signal theory, digital communication over HF channels, machine condition monitoring, signal analysis and classification and information security. The subjects taught at both undergraduate and postgraduate levels includes digital signal processing, advance digital signal processing, and advance digital communications. Assoc. Prof. Dr Ahmad Zuri bin Sha'ameri has conducted short courses for both government and private sectors. At present, he has published 140 papers in his areas of interest at both national and international levels in conferences and journals. 